\title{
Efecto insecticida del polvo de Chenopodium ambrosioides L. y carbonato de calcio en el control de Sitophilus zeamais en granos de maíz
}

\author{
Insecticidal effect of Chenopodium ambrosioides L. powder and calcium carbonate in \\ control of Sitophilus zeamais in corn kernels
}

\author{
Felisa Concepción Gómez ${ }^{1}$, María Bernarda Ramírez ${ }^{2 *}$ y Edgar Francisco Gaona ${ }^{2}$
}

\author{
${ }^{1}$ Departamento de Certificación Fitosanitaria, Dirección de Operaciones, Servicio Nacional de Calidad y Sanidad \\ Vegetal y de Semillas, Asunción, Paraguay. \\ 2 Área de Protección Vegetal, Facultad de Ciencias Agrarias, Universidad Nacional de Asunción, San Lorenzo, \\ Paraguay.
}

* Autor para correspondencia (mramirez@agr.una.py)

Recibido: 01/02/2016; Aceptado: 13/12/2016.

10.18004/investig.agrar.2016.dic ie mbre.116-120

\section{RESUMEN}

El objetivo de la investigación ha sido evaluar la eficacia del polvo vegetal Chenopodium ambrosioides y carbonato de calcio, en concentraciones del 1, 2 y $3 \%$ (p/p), respectivamente, en el control de Sitophilus zeamais Motschulsky en grano de maíz (Zea mays L.). El experimento se ha realizado en el laboratorio de Entomología de la Facultad de Ciencias Agrarias, Universidad Nacional de Asunción (FCA/UNA), ciudad de San Lorenzo, Paraguay; en condiciones controladas de temperatura de $28 \pm 5^{\circ} \mathrm{C}$ y humedad relativa de $70 \pm 5 \%$. El diseño experimental fue completamente al azar con 10 tratamientos y 10 repeticiones con un total de 100 unidades experimentales. Cada unidad experimental fue representada por la concentración de los polvos correspondientes, con 10 insectos adultos en $100 \mathrm{~g}$ de maíz. La variable medida fue mortalidad de $S$. zeamais, evaluadas desde las 24 horas hasta los 55 días después de la aplicación de los polvos. El polvo del C. ambrosioides (Ka'arê) en todas las concentraciones, fue eficaz en el control de $S$. zeamais, desde las 24 horas después de la aplicación (81 a 100\% de mortalidad). Las evaluaciones realizadas con Carbonato de calcio al $90 \%$ en las concentraciones 1 y $2 \%$, respectivamente, demuestran una eficacia de 93 y $94 \%$ de mortalidad a partir de los 15 días después de la aplicación. A los 55 días el carbonato de calcio al $100 \%$ en todas las concentraciones resultó en una eficacia de 98 a $100 \%$ de mortalidad.

Palabras clave: Chenopodium ambrosioides, gorgojo del maíz, control alternativo, polvos vegetales.

\begin{abstract}
The aim of the investigation was to evaluate the efficacy of Chenopodiumambrosioides plant powders and calciu m carbonate at concentrations of 1,2 and $3 \%$ (w/w), respectively in control of Sitophilus zeamais Motschulsky in corn kernels (Zea mays L.). The experiment was conducted in the laboratory of Entomology at Faculty of Agricultural Sciences, National University of Asunción (FCA/UNA), San Lorenzo, Paraguay, under controlled temperature conditions of $28 \pm 5^{\circ} \mathrm{C}$ and relative humidity of $70 \pm 5 \%$. The experimental design was completely randomized with 10 treatments and 10 repetitions with a total of 100 experimental units. Each experimental unit was represented by the concentration of the corresponding powders, with 10 adult insects on $100 \mathrm{~g}$ of corn. The variable measured was mortality of $S$. zeamais evaluated from 24 hours to 55 days after the application of powders. C. ambrosioides powder (Ka'arê) in all concentrations was effective in controlling $S$. zeamais, from 24 hours after application (81 to $100 \%$ mortality). Assessments made with calcium carbonate at $90 \%$ in the concentrations 1 and $2 \%$, respectively, showing and efficiency of 93 and $94 \%$ of mortality fom 15 days after application. At 55 days calcium carbonate at $100 \%$ in all concentrations resulted in an efficacy of 98 and $100 \%$ mortality.
\end{abstract}

Key words: Chenopodium ambrosioides, maize weevil, alternative control, vegetal powder. 


\section{INTRODUCCIÓN}

El maíz es uno de los cereales considerados, mundialmente, como la especie más importante para la alimentación de los seres humanos y animales domésticos, el almacenamiento del grano por largos periodos es esencial para disponer de alimento en forma constante. Entre el 30 y $40 \%$ de la producción de maíz en América latina, se pierde en el almacenamiento. Una de las causas son las plagas de granos almacenados y la de mayor relevancia es el gorgojo del maíz (Sitophilus zeamais Motschulsky, Coleoptera: Curculionidae), ataca en el campo y durante el almacenamiento (Lagunes 1994).

Teniendo en cuenta los perjuicios causados por el insecto plaga $S$. zeamais durante el almacenamiento del maíz, los elevados precios de los defensivos químicos y sus problemas consecuentes del uso inadecuado, es preciso estudiar la eficacia de productos alternativos sobre repelencia y mortalidad del insecto apuntando a minimizar posibles daños a la salud humana (Almeida et al. 2005).

La revalorización de las plantas, como fuente de sustancias con propiedades insecticidas, se viene difundiendo en las últimas décadas. En algunos países de América Latina se han desarrollado interesantes líneas de investigación, buscando en las plantas compuestos químicos con menor impacto ambiental y como potencial en el control de plagas agrícolas (Mazzonetto 2002).

Mezclando el polvo obtenido de plantas con inertes minerales de efecto abrasivo como calcio y talco (carbonato de calcio) disminuyendo las concentraciones del vegetal en polvo en casi un $80 \%$, no altera su alta efectividad y baja toxicidad para mamíferos (Herrera 1995). Los polvos minerales o polvos inertes tienen efecto abrasivo o absorben los lípidos de la epicutícula, facilitando la pérdida de agua que conduce a la muerte de los insectos pordeshidratación (Lucca y Picançao 1995).

Los materiales inertes mezclados con los granos almacenados producen grietas en la cutícula de los insectos asociados, por las cuales se evapora la humedad corporal, lo que ocasiona su deshidratación y muerte (Páez et al. 1990). El uso de plantas y polvos minerales para proteger granos almacenados es una alternativa viable que debe ser investigada y validada con rigor científico debido a que son pocos tóxicos para mamíferos, son de fácil acceso para el agricultor ya que están presentes en su medio como malezas, son de manipulación sencilla, que constituye importantes ventajas especialmente para el productor de escasos recursos (Silva et al. 2003).

El objetivo de esta investigación fue evaluar la eficacia del polvo de Chenopodium ambrosioides y Carbonato de Calcio, en la mortalidad del gorgojo Sitophilus zeamais en granos de maíz.

\section{MATERIALES Y MÉTODOS}

El experimento se llevó a cabo en el laboratorio de Entomología de la Facultad de Ciencias Agrarias de la Universidad Nacional de Asunción (FCA/UNA), ciudad de San Lorenzo, Departamento Central; Paraguay, bajo condiciones controladas de temperatura $28 \pm 5^{\circ} \mathrm{C}$ y una humedad relativa del $70 \pm 5 \%$, y fotoperiodo de 12 hora luz, entre los meses de abril a junio del año 2014.

El material vegetal utilizado fue maíz de la variedad karapé pytã, proveniente del IPTA (Instituto Paraguayo de Tecnología Agraria, Caacupé). Para la desinfestación de los granos, se pesaron previamente $10 \mathrm{~kg}$ de maíz, que fueron depositados en una bandeja de acero inoxidable, luego colocados en una estufa a $60^{\circ} \mathrm{C}$, durante 4 horas, a fin de eliminar los gorgojos en cualquier fase de desarrollo, que pudieran estar presentes en los granos. Una vez terminada la desinfestación se procedió a la selección de granos sanos. Los mismos se limpiaron frotándolos con una tela para la remoción de huevos, que podrían estar adheridos a los granos.

La recolección del Chenopodium ambrosioides se realizó en la etapa de floración, en este estado fenológico las plantas contienen la mayor concentración de compuestos (Mazzonetto 2002). Posteriormente todo el material recolectado fue secado al sol por 48 horas, luego macerado con un mortero de madera. Para confirmar la concentración del polvo del carbonato de calcio al $90 \%$, fue analizado con un equipo de espectrofotometría de absorción atómica, marca Thermo Fisher Scientific, modelo ICE - AA 3300.

Para la cría de insectos se utilizaron ejemplares de la segunda generación, reproducidas en condiciones controladas en el Laboratorio. Los adultos de la primera generación fueron eliminados, para lograr la homogenización de edades de los mismos, dejando en el recipiente solo los granos con huevos. Se dejó transcurrir 10 días para la utilización de nuevos gorgojos. La 
diferenciación de sexos se realizó según criterio de Halstead (1963) quien señala que el rostro de la hembra es evidentemente más largo y menos ornamentado que el macho. Con el estereoscopio fueron observados y separados los gorgojos, luego emparejados para utilizar en las unidades experimentales.

Para la preparación de los tratamientos (Tabla 1) fue utilizada la metodología propuesta por Lagunes y Rodríguez (1989). En un frasco de plástico de $7 \mathrm{~cm}$ de alto y $8 \mathrm{~cm}$ de diámetro con una capacidad de $250 \mathrm{~mL}$, se colocaron $100 \mathrm{~g}$ de maíz agregando los tratamientos respectivos, el polvo vegetal de C. ambrosioides y carbonato de calcio al 90 y $100 \%$ en concentraciones de 1,2 y $3 \%$, respectivamente y un testigo sin aplicación. El grano fue mezclado manualmente con el tratamiento hasta distribuirlo uniformemente. Posteriormente cada frasco o unidad experimental, se infestó con 10 gorgojos (5 machos y 5 hembras) de 10 días de edad, luego cubierta con una tapa previamente perforada para permitir el intercambio gaseoso. Los frascos fueron etiquetados con la información correspondiente a cada tratamiento, $\mathrm{y}$ posteriormente acondicionados a temperatura de $28 \pm 5^{\circ} \mathrm{C}$ y humedad relativa del $70 \pm 5 \%$.

Tabla 1. Tratamientos utilizados en el control de $S$. zeamais en granos de maíz. FCA/UNA. San Lorenzo, 2014.

\begin{tabular}{lc}
\hline \multicolumn{1}{c}{ Tratamientos } & Concentración (\%) /peso de semilla \\
\hline T0 Testigo & Sin C. ambrosioides y carbonato de calcio \\
T1 Polvo de C. ambrosioides & 1 \\
T2 Polvo de C. ambrosioides & 2 \\
T3 Polvo de C. ambrosioides & 3 \\
T4 Polvo de $\mathrm{CaCO}_{3}$ al $90 \%$ & 1 \\
T5 Polvo de $\mathrm{CaCO}_{3}$ al $90 \%$ & 2 \\
T6 Polvo de $\mathrm{CaCO}_{3}$ al $90 \%$ & 3 \\
T7 Polvo de $\mathrm{CaCO}_{3}$ al $100 \%$ & 1 \\
T8 Polvo de $\mathrm{CaCO}_{3}$ al $100 \%$ & 2 \\
T9 Polvo de $\mathrm{CaCO}_{3}$ al $100 \%$ & 3 \\
\hline
\end{tabular}

El diseño experimental fue completamente al azar, con 10 tratamientos y 10 repeticiones cada uno, totalizando 100 unidades experimentales.

Los tratamientos fueron evaluados a las 24, 48, 96, $120 \mathrm{~h}$, luego a los 15 y 55 días, respectivamente, determinándose el porcentaje de mortalidad, el cual fue corregido por la fórmula de Abbott (1925).

$$
\mathrm{MC}=\frac{\mathrm{MTr}-\mathrm{MTe}}{100-\mathrm{MTe}} \times 100
$$

Donde:

MC $=$ Porcentaje Mortalidad Corregida

Mtr $=$ Porcentaje Mortalidad en el tratamiento

Mte $=$ Porcentaje Mortalidad en el testigo absoluto

Se considera insecto muerto cuando no tiene movilidad locomotora o presente movimientos descoordinados tendientes a la muerte.

Los datos obtenidos fueron registrados en planillas para su posterior análisis de varianza utilizando el software estadístico InfoStat, versión 2014 (Di Rienzo et al. 2014) y las medias fueron comparadas por el test de Tukey al $5 \%$.

\section{RESULTADOS Y DISCUSIÓN}

En la primera evaluación, a las 24 horas después de la aplicación de los productos (DAP), se pudo constatar mortalidades de 81,100 y $100 \%$ de S. zeamais con los tratamientos $\mathrm{T} 1 \quad(C$. ambrosioides 1\%), $\mathrm{T} 2 \quad(C$. ambrosioides 2\%) y $\mathrm{T} 3$ (C. ambrosioides 3\%), respectivamente, siendo $\mathrm{T} 2$ y $\mathrm{T} 3$, estadísticamente similares entre sí y distintos al T1. Por otro lado, los tres tratamientos fueron diferentes al testigo, en el cual se observó el $100 \%$ de sobrevivencia de S. zeamais.

A las 48 horas (DAP) aumentó la mortalidad de $S$. zeamais a $99 \%$ en el tratamiento $\mathrm{T} 1$, sin embargo en los demás tratamientos $\mathrm{T} 4$ (Polvo de $\mathrm{CaCO}_{3}$ al $90 \%$ en concentración 1\%), T5 (Polvo de $\mathrm{CaCO}_{3}$ al $90 \%$ en concentración 2\%) T6 (Polvo de $\mathrm{CaCO}_{3}$ al $90 \%$ en concentración 3\%), T7 (Polvo de $\mathrm{CaCO}_{3}$ al $100 \%$ en 
concentración 1\%), T8 (Polvo de $\mathrm{CaCO}_{3}$ al $100 \%$ en concentración 2\%) y $\mathrm{T} 9$ (Polvo de $\mathrm{CaCO}_{3}$ al $100 \%$ en concentración $3 \%$ ) con una mortalidad muy baja de 9 a $45 \%$. El testigo se mantuvo constante $(100 \%$ de sobrevivencia del insecto). A las 96 horas DAP, en el tratamiento T1 se observó el $100 \%$ de mortalidad de los insectos, seguido del T7 con 70\% de mortalidad (Tabla 2). A las 120 horas DAP se constató que los tratamientos T4 (Polvo de $\mathrm{CaCO} 3$ al $90 \%$ en concentración 1\%), T5 (Polvo de $\mathrm{CaCO} 3$ al $90 \%$ en concentración 2\%) y $\mathrm{T} 7$ (Polvo de $\mathrm{CaCO} 3$ al $100 \%$ en concentración 1\%) demostraron mortalidades de 81,72 y $95 \%$ respectivamente, siendo superior estadísticamente el T7 (Polvo de $\mathrm{CaCO} 3$ al $100 \%$ en concentración 1\%), y similares entre sí a los 15 días pero diferentes al testigo
(3\% de mortalidad). A los 55 días de haberse realizado el experimento todos los tratamientos presentaron eficacia, estadísticamente fueron similares entre sí, pero diferentes con respecto al testigo (Tabla 2). Trascurrido el mismo periodo de tiempo, los tratamientos $\mathrm{T} 4$ (Polvo de $\mathrm{CaCO} 3$ al $90 \%$ en concentración 1\%), T5 (Polvo de $\mathrm{CaCO} 3$ al $90 \%$ en concentración 2\%) y $\mathrm{T} 7$ (Polvo de $\mathrm{CaCO} 3$ al $100 \%$ en concentración 1\%) presentaron $100 \%$ de eficacia, luego le siguen los tratamientos T6 (Polvo de CaCO3 al 90\% en concentración 3\%), T8 (Polvo de $\mathrm{CaCO} 3$ al $100 \%$ en concentración 2\%) y T9 (Polvo de $\mathrm{CaCO} 3$ al $100 \%$ en concentración 3\%) con una mortalidad de 89, 98 y 99\%. Todos los tratamientos fueron similares entre sí, pero diferentes al testigo.

Tabla 2. Porcentaje de mortalidad de adultos de $S$. zeamais, en diferentes concentraciones $d e \quad C$. ambrosioides y Carbonato de Calcio en granos de maíz. FCA/UNA, San Lorenzo, 2014.

\begin{tabular}{lcccccc}
\hline Tratamiento & 24 horas & $\mathbf{4 8}$ horas & $\mathbf{9 6}$ horas & $\mathbf{1 2 0}$ horas & $\mathbf{1 5}$ días & $\mathbf{5 5}$ días \\
\hline T0 & $0,00 \mathrm{~d}^{*}$ & $0.00 \mathrm{~d}$ & $0,00 \mathrm{e}$ & $0,00 \mathrm{e}$ & $3,00 \mathrm{~d}$ & $18.00 \mathrm{~b}$ \\
T1 & $81,00 \mathrm{~b}$ & $99,000 \mathrm{a}$ & $100,00 \mathrm{a}$ & $100,00 \mathrm{a}$ & $100,00 \mathrm{a}$ & $100,00 \mathrm{a}$ \\
T2 & $100,00 \mathrm{a}$ & $100,00 \mathrm{a}$ & $100,00 \mathrm{a}$ & $100,00 \mathrm{a}$ & $100,00 \mathrm{a}$ & $100,00 \mathrm{a}$ \\
T3 & $100.00 \mathrm{a}$ & $100,00 \mathrm{a}$ & $100,00 \mathrm{a}$ & $100,00 \mathrm{a}$ & $100.00 \mathrm{a}$ & $100,00 \mathrm{a}$ \\
T4 & $6,00 \mathrm{~cd}$ & $15,00 \mathrm{c}$ & $66,00 \mathrm{~b}$ & $81,00 \mathrm{c}$ & $93,00 \mathrm{a}$ & $100,00 \mathrm{a}$ \\
T5 & $10,00 \mathrm{~cd}$ & $19,00 \mathrm{c}$ & $69,00 \mathrm{~b}$ & $72,00 \mathrm{c}$ & $94,00 \mathrm{a}$ & $100,00 \mathrm{a}$ \\
T6 & $7,00 \mathrm{~cd}$ & $12,00 \mathrm{c}$ & $20,00 \mathrm{c}$ & $38,00 \mathrm{~d}$ & $38,00 \mathrm{bc}$ & $89,00 \mathrm{a}$ \\
T7 & $8,00 \mathrm{~cd}$ & $45,00 \mathrm{~b}$ & $70,00 \mathrm{~b}$ & $95,00 \mathrm{ab}$ & $100,00 \mathrm{a}$ & $100,00 \mathrm{a}$ \\
T8 & $3,00 \mathrm{~d}$ & $9,00 \mathrm{~cd}$ & $10,00 \mathrm{~cd}$ & $27,00 \mathrm{~d}$ & $27,00 \mathrm{c}$ & $8,00 \mathrm{a}$ \\
T9 & $19,31 \mathrm{c}$ & $19,00 \mathrm{c}$ & $19,00 \mathrm{c}$ & $41,00 \mathrm{~d}$ & $41,31 \mathrm{~b}$ & $99,00 \mathrm{a}$ \\
\hline CV (\%) & 29,78 & 18,31 & 21,09 & 15,40 & 12,83 & 9,94 \\
\hline Tratamientos con igual letra, en la columna, no difieren estadísticamente por el test deTukey $(\alpha=0,05)$.
\end{tabular}

Los resultados del presente estudio son similares al de Procopio et al. (2003) quienes utilizando C. ambrosioides a una concentración de $3 \%$ obtuvieron $100 \%$ de mortalidad de S. zeamais. También Silva et al. (2003) demostraron una mortalidad de $100 \%$ de $S$. zeamais utilizando C. ambrosioides al 1\%. Estudios realizados por Tavares (2002) ha comprobado que la mortalidad de adultos con polvos de inflorescencia de C. ambrosioides presentan mejores resultados que polvos de mezcla de las hojas y tallos, se debe a una mayor concentración de los semioquímicos que tienen un efecto insecticida que se encuentra en estos órganos. Silva et al. (2004) mencionan que el carbonato de calcio $2 \%$, a los 30 días de aplicación se observa una alta eficacia, llegando a $100 \%$ de mortalidad de $S$. zeamais.

\section{CONCLUSIONES}

El polvo de C. ambrosioides es eficaz en todas las concentraciones en el control de S. zeamais desde las 24 
horas. El Carbonato de calcio al 90 y $100 \%$, presentan una eficacia de 89 a $100 \%$ de mortalidad a los 55 días.

\section{REFERENCIAS BIBLIOGRAFICAS}

Abbott, WS. 1925. A method for computing the effectiveness of na insecticide. Journal of Economic Entomology 18:265-267.

Almeida, FAC; Almeida, SA; Santos, NR. 2005. Efeitos de extratos alcoólicos de plantas sobre o caruncho do feijão vigna (Callosobruchus maculatus). Revista Brasileira de Engenharia Agrícola e Ambiental. 9(4):585-590.

Di Rienzo, JA; Casanoves, F; Balzarini, MG; González, L; Tablada, M; Robledo, CW. 2014. InfoStat versión 2014 (en línea). Córdoba, Argentina, Grupo Info Stat, Facultad de Ciencias Agrarias, Universidad Nacional de Córdoba. Consultado el 10 dic. 2015. Disponible en http://www.infostat.com.ar

Halstead, DGH. 1963. External sex differences in storedproducts (Coleoptera) (en línea). Bulletin of Entomological Research 54:119-134. Consultado el 20 jun. 2015. Disponible en http://www.scielo.cl/scielo. php? script $=$ sci_nlinks \&ref=278213\&pid $=$ S0365-2807 $200700020000400006 \& \ln \mathrm{g}=\mathrm{es}$

Herrera, JJR. 1995. Combinación de tres polvos vegetales y dos minerales con propiedades insecticidas para el combate del gorgojo del maíz Sitophilus zeamais Motschulsky en maíz almacenado. Tesis Ing. Agr. Córdoba, Argenita, Facultad de Ciencias Agrícolas, Universidad Veracruzana. $83 \mathrm{p}$.

Lagunes, TA; C. Rodríguez H. 1989. Búsqueda de tecnología apropiada para el combate de plagas del maíz almacenado en condiciones rústicas. Montecillo, México, CONACYT/Colegio de Postgraduados. 150 p.

Lagunes, TA. 1994. Extractos, polvos vegetales y polvos minerales para el combate de plagas del maíz y del frijol en la agricultura de subsistencia. México: Colegio de Postgraduados/USAID/CONACYT /BORUCONSA. $32 \mathrm{p}$.
Lucca, A; Picançao, M. 1995. Manejo integrado de pragas do feijoeiro no armazenamento. Rev. Brasileira de Armazenamento 20:37-43.

Mazzonetto, F. 2002. Efeito de genotipos de feijoeiro e de pós de origem vegetal sobre Zabrotes subfasciatus (Boh.) e Acanthoscelides obtectus (Say) (Coleoptera: Bruchidae). Tese Dr. Piracicaba, Brasil, Universidade de São Paulo. 134 p.

Páez, A; Lagunes, A. 1990. Polvos vegetales y materiales inertes para el combate del gorgojo Sitophilus zeamais (Coleoptera: Curculionidae) en maíz almacenado. Agrociencia 1:35-46.

Procopio, S; Vendramim, J; Ribeiro, J; Santos, JB. 2003. Efeito de pós vegetales sobre Acanthoscelides obctetus (SAY) e Zabrotes subfasciatus (Boh) (Coleóptero: Bruchidae). Revista Ceres 50:395-405.

Silva, G; Lagunes, A. Rodríguez, J. 2003. Control de Sitophilus zeamais (Coleoptera:Curculionidae) con polvos vegetales solos y en mezcla con carbonato de calcio en maíz almacenado. Cien. Inv. Agr. 30:153160.

Silva, G; González-Gómez, P; Hepp Gallo, R; Casls Bustos, P. 2004. Control de Sitophilus zeamais Motschulsky con polvos inertes. Agrociencia 38(5):529536.

Tavares, MAGC. 2002. Bioatividade da-erva-de Santa María, Chenopodium ambrosioides L. (Chenopodiaceae), en relación a Sitophilus zeamais (Coleoptera: Curculionidae), Tesis Msc, Piracicaba, Brasil, Universidad de São Paulo. 59 p. 